\title{
Changes in Infants Faecal Characteristics and Microbiota by Inulin Supplementation
}

\author{
Wendy K.W. Yap', Suhaila Mohamed ${ }^{1, *}$, Mohammad Husni Jamal', Meyer Diederick², \\ and Yazid A. Manap ${ }^{1}$ \\ ${ }^{I}$ Functional Food Laboratory, Faculty of Food Science and Technology, Universiti Putra Malaysia, \\ 43400, Serdang, Selangor, Malaysia \\ ${ }^{2}$ Sensus, PO Box 1308, 4700 BH Roosendaal, Netherlands
}

Received 25 December, 2007; Accepted 18 April, 2008

\begin{abstract}
Summary The effects of inulin on the microbial composition and faecal characteristics in 36 healthy, formula-fed infants (average age 7.7 months) given 3 different daily dosages of native inulin (0.75 g/day, $1.00 \mathrm{~g} / \mathrm{day}$, and $1.25 \mathrm{~g} / \mathrm{day})$ were studied. At all levels of inulin consumption, a significant $(p<0.05)$ reduction of potential pathogenic microorganisms such as clostridia was found. An intake of $1.25 \mathrm{~g} / \mathrm{day}$ of inulin caused a significant $(p<0.05)$ increase of Bifidobacterium spp. as well as a significant $(p<0.05)$ decline in Gram-positive cocci and coliform bacteria. Inulin consumption resulted in a significant $(p<0.05)$ decrease in faecal $\mathrm{pH}$ value and changes in faecal weight, faecal texture and colour, indicating improvement in healthy bile production and bacterial fermentation. It is concluded that inulin consumption in formula-fed infants after weaning positively affected the microbial composition of faeces and faecal properties.
\end{abstract}

Key Words: inulin, stool properties, microbiota, formula-fed, infants

\section{Introduction}

The $\beta$-(2-1)-fructans inulin and oligofructose or fructooligosaccharides (FOS), are natural carbohydrates, found in many foods such as leek, garlic, onion, artichoke, chicory, banana, asparagus and wheat $[1,2]$. Based on consumption data, the average intake of inulin and FOS in a normal human diet has been estimated at about $2-10 \mathrm{~g}$ per day $[1,3]$.

These carbohydrates resist hydrolysis and digestion in stomach and small intestine, and thus reach the colon intact. There they are fermented by the colonic microbiota to short chain fatty acids (SCFA) and gas. Selective fermentation of these fructans by bifidobacteria can result in an improved

\footnotetext{
*To whom correspondence should be addressed

Tel: +60-3-8946-8391 Fax: +60-3-8942-3552

E-mail: mohamed.suhaila@gmail.com
}

composition of gut microflora. This so-called prebiotic effect has been found in many human studies with adult volunteers [4] and with different types of inulin [5-9]. In vitro studies also showed that oligofructose and inulin selectively stimulated the growth of bifidobacteria [10].

FOS and inulin not only exert local colonic physiological effects, but also systemic effects, via the absorption of their fermentation products (SCFA) from the colon. The local effects of inulin consumption comprise the increased synthesis of vitamins, an increase in mineral, especially of calcium absorption from the colon [11], and possibly a lowered risk for colon cancer [12]. The systemic effects include a lowered level of serum lipids [13], an increased feeling of satiety and decreased energy intake $[14,15]$, or the modulation of immune functions [16].

Most of these prebiotic studies have been carried out with adult volunteers, but the potential physiological benefits are equally important for infants. Not surprisingly therefore 
prebiotics studies are also being conducted in younger people. Changes in composition of the colonic microbiota [17] and the possible health benefits of a mixture of galactooligosaccharides and inulin in formula-fed babies were reported [18]. Native inulin reportedly has a prebiotic effect in formula-fed babies [19]. However, very little work if at all has been reported on the effects of native inulin in infants of older ages (past weaning) and the lowest dose that will give a demonstrable physiological effect in infants. The objective of this study is to investigate the effect of 3 different levels $(0.75 \mathrm{~g} / \mathrm{day} ; 1.00 \mathrm{~g} /$ day and $1.25 \mathrm{~g} /$ day $)$ of native inulin supplements on the composition of the colonic microbiota and their effects on stool characteristics in children aged about 8 months.

\section{Materials and Methods}

\section{Subjects}

Healthy, free-living, 5-12 months old infants from Serdang, who had undergone no antibiotic treatments during the previous three months, participated in the study. The parents of the infants ( 23 girls and 13 boys, with an average age of $8.1 \pm 1.6$ months; average weight $7.9 \pm 1.4 \mathrm{~kg}$ and average height of $68.0 \pm 3.8 \mathrm{~cm}$ at the time of study) were fully informed both orally and in written form about the aims of the study and the procedures. The children were all past weaning age. The protocol was approved by the Ethics Committee of Faculty of Medicine, Universiti Putra Malaysia (Ref. no: UPM/FPSK/100-11/40, dated 10 October 2001) and informed consent of the parents was obtained.

\section{Experimental design}

All the participating subjects were fully supplied with control diet (consisting of a follow-up infant formula, different types of Nestum ${ }^{\circledR}$ baby food, biscuits), disposable nappies and other facilities such as medical services throughout the study. The 36 subjects were randomly assigned to receive three levels of inulin supplementation, added into their bottle of infant formula during preparation, at the specified dose. Native inulin (Frutafit ${ }^{\circledR}$ IQ) with average degree of polymerization of 9 was supplied by Sensus, Netherlands.

The children were subjected to seven days of basal diet, followed by either $0 \mathrm{~g} /$ day $(n=8), 0.75 \mathrm{~g} /$ day $(n=10$, Group I), $1.0 \mathrm{~g} /$ day ( $n=9$, Group II) and $1.25 \mathrm{~g} /$ day $(n=9$, Group III) of inulin for fourteen days and another fourteen days of washout period. The total number of days for each trial is 35 days.

\section{Assessment of dietary intake and stool condition}

Throughout the study, the parents of the subjects were required to complete a daily dietary recall questionnaire, which provided information about dietary pattern, possible intestinal discomfort (flatulence, soft stools or diarrhoea) as well as frequency of defecation and appearance of stools. The consistency of the stools were classified into 5 categories i.e. watery, soft, muddy, hard cylindrical and very hard pellets and their colours were classified into 3 categories greenish or yellowish, golden (light or dark), and brown (brownish or dark).

\section{Sample collection}

The last three days of each treatment fresh stools were collected ( $72 \mathrm{~h}$ collection) i.e. during the basal period, during the supplementation period and during the washout period. The stool sample was taken after mixing all stools from the $72 \mathrm{~h}$ collection.

\section{Bacteriological analyses (modified [7])}

All fresh faeces samples were transported back to the laboratory anaerobically at below $10^{\circ} \mathrm{C}$ within $60 \mathrm{~min}$. Samples were weighed and $\mathrm{pH}$ was measured in a $10 \%$ (w/w) suspension in demineralised water. A sample was homogenized in $0.1 \mathrm{~mol} / 1$ ( $\mathrm{pH} 7.0)$ potassium phosphate buffer to obtain $0.1 \mathrm{~g} / \mathrm{ml}$ faecal suspension. Serial dilutions $\left(10^{-2}\right.$ to $\left.10^{-11}\right)$ were prepared using half strength peptone water (Merck, Darmstadt, Germany). An aliquot of $0.1 \mathrm{ml}$ of each dilution was spread plated (in duplicate) onto agar medium specific for the growth of total anaerobes (WilkinsChalgren anaerobic agar, Oxoid-Unipath Ltd., Basingstoke, Hampshire, England), bifidobacteria (Modified TrypticasePhytone-Yeast Extract agar, Scharlau, Spain), lactobacilli (Rogosa SL agar, Merck), coliforms (MacConkey \#2, Oxoid-Unipath Ltd.), clostridia (Reinforced Clostridia agar, Pronadisa, Madrid, Spain), Gram-positive cocci (Azide Blood agar, Pronadisa) and total aerobes (Nutrient agar, Merck). For recovery of anaerobic bacteria (bifidobacteria, clostridia, coliforms, lactobacilli, total anaerobes and Gram-positive cocci) the plates were incubated at $37^{\circ} \mathrm{C}$ for three days. Anaerobic conditions were achieved by using anaerobic jars containing Anaerocult A (Merck). For recovery of total aerobes, the plates were incubated at $37^{\circ} \mathrm{C}$ for $24 \mathrm{~h}$. All plates having 30 to 300 colonies were counted on a Quebec colony counter (Acolyte, Yorkshire, England).

\section{Preparation of stock cultures of bifidobacteria}

Ten percent of each type of colony of the bifidobacteria plates (Modified Trypticase-Phytone-Yeast Extract agar, TPY) was picked up and Gram-stained for morphological observation. The colonies were purified by repeatedly streaking on modified TPY agar. Samples of single colonies thus obtained were subcultured twice in TPY broth at $37^{\circ} \mathrm{C}$ for $18 \mathrm{~h}$ under anaerobic conditions. The cultures were then subjected to centrifugation at $3000 \mathrm{rpm}$ for $10 \mathrm{~min}$. The supernatant was discarded. The precipitate was mixed with $1 \mathrm{ml}$ of $90 \% \mathrm{v} / \mathrm{v}$ glycerol in $10 \% \mathrm{w} / \mathrm{v}$ TPY broth and stored 
at $-20^{\circ} \mathrm{C}$ until further use.

\section{Identification of bifidobacteria}

For recovery of stock cultures, the microorganisms were grown in TPY broth anaerobically at $37^{\circ} \mathrm{C}$ for $48 \mathrm{~h}$ or more until sufficient growth was observed. The cultures were again subcultured twice before analysis. Gram-staining and morphological observation was performed according to a standard method [20]. The carbohydrate fermentation profile was analyzed using a commercial API 20A Kit (Biomerieux, Marcy-1'Etoile, France) for identification of Bifidobacterium spp. The reactions were observed after 24-48 h of incubation at $37^{\circ} \mathrm{C}$. Identification was obtained by referring to the identification table in the API 20A Analytical Profile Index Manual (APILAB).

\section{Statistical analysis}

Data were expressed as mean \pm standard deviation and one-way Analysis of Variance (ANOVA) was performed using MINITAB version 14 (MINITAB Inc., State College, PA). Statistical significance was accepted at a probability level of $p<0.05$.

\section{Results}

\section{Faecal flora}

Table 1 summarizes the microbial composition of the faecal samples during the various treatment periods. All three groups of subjects had similar numbers of total anaerobes (about $10^{10} \mathrm{cfu} / \mathrm{g}$ faeces) during the basal period. This number was not affected by the intake of 0.75 or $1.0 \mathrm{~g} /$ $\mathrm{d}$ of inulin, but decreased significantly $(p<0.05)$ with $1.25 \mathrm{~g} /$ $\mathrm{d}$ of inulin consumption. The total number of anaerobes came back to the original value during the washout period of the three groups of inulin-supplemented infants. The total aerobe plate counts in the present study did not show any

Table 1. Faecal microbial composition during various treatment periods

\begin{tabular}{|c|c|c|c|c|}
\hline \multirow{2}{*}{ Bacteria } & \multirow{2}{*}{ Group } & \multicolumn{3}{|c|}{ Bacteria in $\log ^{10} \mathrm{cfu} / \mathrm{g}( \pm \mathrm{SD})$ during } \\
\hline & & Basal period & Inulin period & Washout period \\
\hline \multirow[t]{4}{*}{ Total anaerobes } & $\mathrm{I}(n=10)$ & $10.57 \pm 0.35$ & $10.73 \pm 0.29$ & $10.15 \pm 0.44$ \\
\hline & II $(n=9)$ & $10.34 \pm 0.18$ & $9.89 \pm 0.83$ & $10.50 \pm 0.25$ \\
\hline & III $(n=9)$ & $10.28 \pm 0.35$ & $9.67 \pm 0.30^{*}$ & $10.10 \pm 0.12$ \\
\hline & $\operatorname{IV}(n=8)$ & $10.45 \pm 0.35$ & $10.46 \pm 0.30$ & $10.43 \pm 0.35$ \\
\hline \multirow[t]{4}{*}{ Bifidobacteria } & $\mathrm{I}(n=10)$ & $9.16 \pm 0.57$ & $9.36 \pm 0.58$ & $8.91 \pm 0.51$ \\
\hline & II $(n=9)$ & $9.64 \pm 0.28$ & $9.77 \pm 0.38$ & $9.83 \pm 0.26$ \\
\hline & III $(n=9)$ & $9.23 \pm 0.38$ & $9.55 \pm 0.17 *$ & $9.56 \pm 0.34$ \\
\hline & $\operatorname{IV}(n=8)$ & $9.05 \pm 0.41$ & $9.04 \pm 0.32$ & $9.32 \pm 0.39$ \\
\hline \multirow[t]{4}{*}{ Lactobacilli } & $\mathrm{I}(n=10)$ & $8.61 \pm 0.93$ & $8.90 \pm 0.68$ & $8.70 \pm 0.56$ \\
\hline & II $(n=9)$ & $9.61 \pm 0.15$ & $8.86 \pm 0.15^{*}$ & $9.31 \pm 0.19$ \\
\hline & III $(n=9)$ & $8.78 \pm 0.43$ & $8.15 \pm 0.75$ & $8.20 \pm 0.71$ \\
\hline & $\operatorname{IV}(n=8)$ & $5.92 \pm 0.51$ & $5.01 \pm 0.52$ & $6.13 \pm 0.43$ \\
\hline \multirow[t]{4}{*}{ Coliforms } & $\mathrm{I}(n=10)$ & $8.51 \pm 0.38$ & $8.44 \pm 0.58$ & $9.23 \pm 0.61$ \\
\hline & $\mathrm{II}(n=9)$ & $8.85 \pm 0.69$ & $8.50 \pm 0.46$ & $8.79 \pm 0.47$ \\
\hline & III $(n=9)$ & $9.68 \pm 0.32$ & $8.13 \pm 0.40^{*}$ & $8.80 \pm 0.49$ \\
\hline & $\operatorname{IV}(n=8)$ & $9.22 \pm 0.35$ & $9.26 \pm 0.52$ & $9.41 \pm 0.56$ \\
\hline \multirow[t]{4}{*}{ Clostridia } & $\mathrm{I}(n=10)$ & $10.15 \pm 0.15$ & $9.28 \pm 0.35^{*}$ & $9.34+0.55$ \\
\hline & II $(n=9)$ & $9.98 \pm 0.32$ & $8.95 \pm 0.20^{*}$ & $10.20+0.35$ \\
\hline & III $(n=9)$ & $9.66 \pm 0.32$ & $8.78 \pm 0.39 *$ & $9.59+0.25$ \\
\hline & $\operatorname{IV}(n=8)$ & $9.87 \pm 0.24$ & $9.88 \pm 0.23$ & $9.89 \pm 0.25$ \\
\hline \multirow[t]{4}{*}{ Gram-positive cocci } & $\mathrm{I}(n=10)$ & $9.76 \pm 0.71$ & $8.74 \pm 0.55^{*}$ & $8.78 \pm 0.55$ \\
\hline & II $(n=9)$ & $9.38 \pm 0.62$ & $8.88 \pm 0.27$ & $10.04 \pm 0.42$ \\
\hline & III $(n=9)$ & $9.12 \pm 0.42$ & $8.42 \pm 0.28^{*}$ & $9.10 \pm 0.67$ \\
\hline & $\operatorname{IV}(n=8)$ & $9.05 \pm 0.53$ & $9.21 \pm 0.44$ & $9.66 \pm 0.59$ \\
\hline
\end{tabular}

Group I: $0.75 \mathrm{~g} / \mathrm{d}$ of inulin during supplementation, Group II: $1.00 \mathrm{~g} / \mathrm{d}$ of inulin; Group III: $1.25 \mathrm{~g} / \mathrm{d}$ of inulin. Group IV: $0 \mathrm{~g} / \mathrm{d}$ of inulin. Each group serve as their own control. Significant differences between groups for lactobacillus and bifidobacteria at basal period, were unexpected, since the babies were distributed randomly without any prior knowledge of their faecal microbiota profile. All babies were from the same area of residence.

*Significant changes as compared to basal period $(p<0.05)$ during inulin supplementation 
significant changes $(p>0.05)$ during any of the treatments (not shown).

All levels of inulin supplementation had a significant effect $(p<0.05)$ on the numbers of clostridia. The initial numbers of clostridia in the studied groups were high $\left(10^{10} \mathrm{cfu} / \mathrm{g}\right.$ faeces) during basal diet and were reduced with almost 1 log unit during different levels of inulin supplementation.

The distribution of Gram-positive cocci in the beginning of basal period remained at $10^{9} \mathrm{cfu} / \mathrm{g}$ faeces, but showed a significant reduction $(p<0.05)$ during both $0.75 \mathrm{~g} / \mathrm{d}$ and $1.25 \mathrm{~g} / \mathrm{d}$ inulin consumption whereas no significant difference $(p>0.05)$ was seen with $1.0 \mathrm{~g} / \mathrm{d}$ inulin intake. The total number of coliforms amounted to $10^{8}-10^{9} \mathrm{cfu} / \mathrm{g}$ faeces. Only a slight decrease of coliforms was found with $0.75 \mathrm{~g} / \mathrm{d}$ and
$1.00 \mathrm{~g} / \mathrm{d}$ of inulin supplementation whereas a significant decrease $(p<0.05)$ in coliforms was shown in the $1.25 \mathrm{~g} / \mathrm{d}$ inulin supplementation.

The numbers of bifidobacteria during inulin supplementation showed a non-significant increase with both $0.75 \mathrm{~g} / \mathrm{d}$ and $1.00 \mathrm{~g} / \mathrm{d}$ of inulin intake, whereas with $1.25 \mathrm{~g} / \mathrm{d}$ of inulin a significant increase $(p<0.05)$ in bifidobacteria numbers was found. At this inulin consumption the magnitude of the bifidogenic effect seemed dependent on the starting number of bifidobacteria (Fig. 1).

In the present study no stimulation of Lactobacillus growth was found. The numbers of lactobacilli isolated from the fresh faeces were in the range of $10^{8}-10^{9} \mathrm{cfu} / \mathrm{g}$ in most of the infants. Only $1 \mathrm{~g} / \mathrm{d}$ inulin showed a significant decrease in lactobacilli bacterial count. In the control group of 8

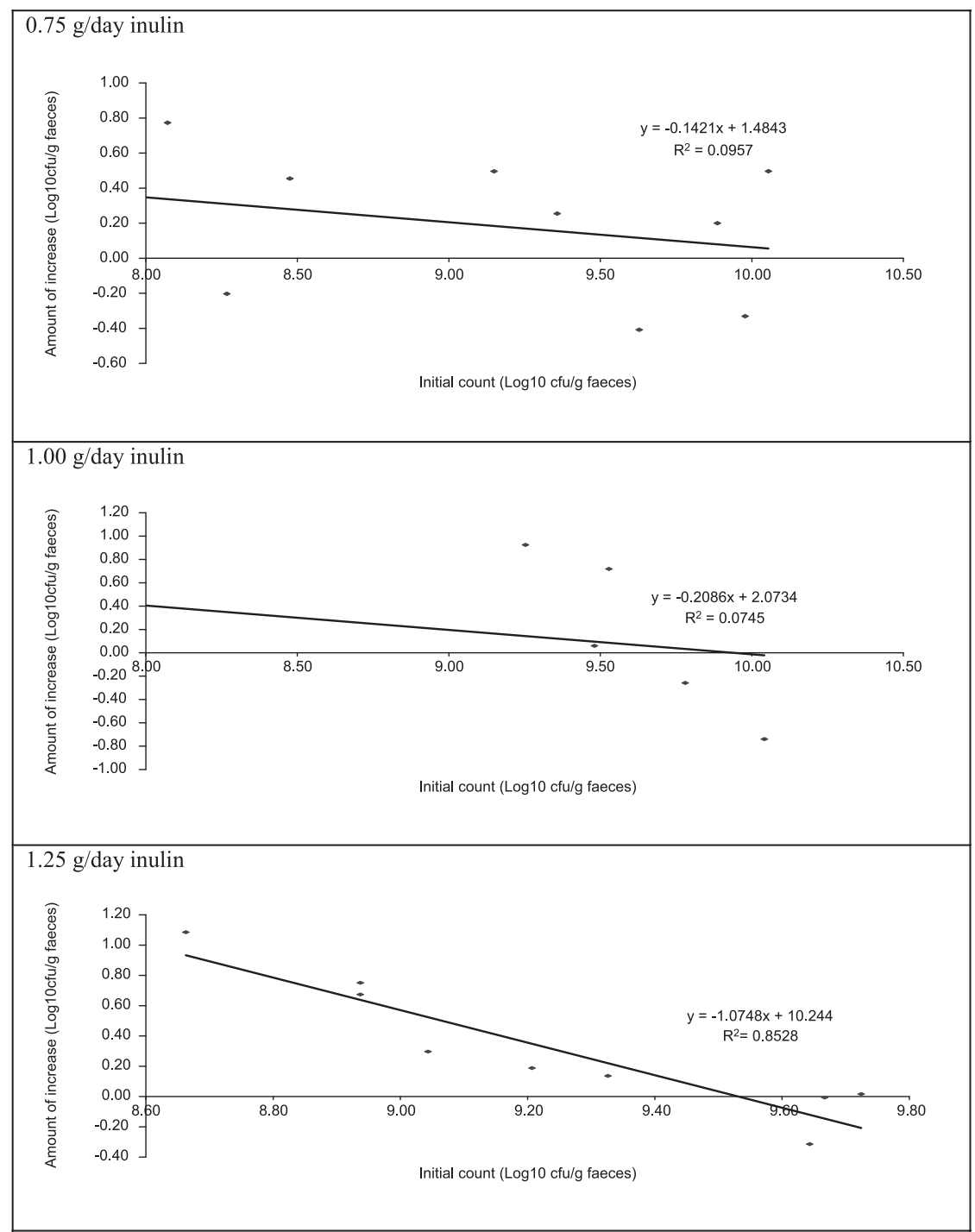

Fig. 1. Increase in Bifidobacterium sp. as a function of the initial number of Bifidobacterium sp. at various levels of inulin supplementation. The faecal content of Bifidobacterium sp. was determined with and without inulin supplementation of the diet, and the logarithmic increase was calculated. The line is the best fit as calculated (by Excel). 
infants that did not receive inulin treatment no changes in the microbial profile were detected during the 35 days.

\section{Identification of Bifidobacterium isolates}

The morphology of the colonies on TPY varied from convex to lens-shaped, or convex to pulvinate. Their colour and appearance varied from opaque to shining and from porcelain white through whitish to cream, with an undulating or smooth to mucoid-soft surface. The morphology of the bacteria was found to be bifurcated $\mathrm{Y}$ and $\mathrm{V}$ rodshaped as observed under phase contrast microscopy. All isolates were Gram-positive (blue violet to Gram-stain). Among 78 isolates isolated from faeces collected during the basal period without inulin, thirty six isolates were identified as follows based on their carbohydrate fermentation pattern: 18\% Bifidobacterium bifidum strain 1 (Bb 1), 64\% Bifidobacterium bifidum strain $2\left(\begin{array}{lll}B b & 2\end{array}\right)$ and $18 \%$ unidentified genus under the Actinomyces grouping. The unidentified isolates and Bifidobacterium bifidum strain 1 ( $B b 1)$ were differentiated by their ability to ferment arabinose.

The isolates from the periods with inulin-supplemented diet were identified as follows: 7.7\% $\mathrm{Bb} 1$ and 92.3\% $\mathrm{Bb} 2$. Apparently, inulin consumption leads to a shift in species. Isolates that were able to ferment arabinose were identified as Bifidobacterium bifidum strain 1 with $97.5 \%$ of identification. $B b 1$ or $B b 2$ are the two different strains of $B$. bifidum identified through various biochemical tests.

\section{Stool characteristics}

The changes in stool characteristics in terms of $\mathrm{pH}$, consistency, frequency and weight are presented in Table 2. Inulin ingestion led to a significant $(p<0.05)$ reduction of the faecal $\mathrm{pH}$ in the two highest dosages, in a dose dependent manner. Changes in faecal frequency and mass were also observed during inulin supplementation, but these effects did not reach statistical significance.

The consistency of the stools changed from being soft, muddy and hard cylindrical during the basal period, to mostly soft during the three levels of inulin supplementation as indicated by the lower numbers in Table 2. However none of these changes reached statistical significance. Changes in stool colour were found with inulin supplementation, whereby the percentage number of yellow/green and lighter coloured stools decreased and the percentage of brown or darker coloured stools increased compared to basal treatment (Fig. 2). Infants treated with $0.75 \mathrm{~g} /$ day inulin showed an increase in brown stools and a reduction in yellow/ greenish stool during treatment period. During the washout period, the number of brown stools decreased, while that of the yellow/greenish stool increased again. Infants treated with $1.00 \mathrm{~g} /$ day inulin also showed increase in the number of brown stools and a reduction in yellow/greenish stool during treatment. Again, during the washout period, the number of brown stools decreased, while that of the yellow/greenish stool increased. Infants treated with $1.25 \mathrm{~g} / \mathrm{day}$ inulin showed no significant change in stools colour during treatment, followed by a significant increase in brown stools and

Table 2. Faecal characteristics

\begin{tabular}{llccc}
\hline Parameter & Group & Basal period & Inulin period & Washout period \\
\hline Faecal pH & I $(n=10)$ & $6.8 \pm 0.5$ & $6.7 \pm 0.6$ & $6.9 \pm 0.7$ \\
& II $(n=9)$ & $6.8 \pm 0.6$ & $6.3 \pm 0.5^{*}$ & $6.6 \pm 0.4$ \\
& III $(n=9)$ & $6.7 \pm 0.7$ & $5.9 \pm 0.3^{*}$ & $6.4 \pm 0.6$ \\
Defecation frequency & IV $(n=8)$ & $7.1 \pm 0.6$ & $7.2 \pm 0.4$ & $7.4 \pm 0.5$ \\
(per day) & I $(n=10)$ & $1.07 \pm 0.25$ & $1.13 \pm 0.25$ & $1.33 \pm 0.15$ \\
& II $(n=9)$ & $1.28 \pm 0.13$ & $1.22 \pm 0.18$ & $1.17 \pm 0.17$ \\
Faecal mass (g/day) & III $(n=9)$ & $1.56 \pm 0.17$ & $1.48 \pm 0.15$ & $1.52 \pm 0.15$ \\
& IV $(n=8)$ & $1.11 \pm 0.19$ & $1.12 \pm 0.20$ & $1.19 \pm 0.23$ \\
& I $(n=10)$ & $43.9 \pm 12.8$ & $55.8 \pm 14.8$ & $57.2 \pm 12.8$ \\
Faecal consistency & II $(n=9)$ & $44.2 \pm 11.6$ & $49.7 \pm 13.8$ & $48.5 \pm 10.6$ \\
[scored from 1: & III $(n=9)$ & $54.3 \pm 14.1$ & $58.0 \pm 11.4$ & $51.3 \pm 14.1$ \\
watery-5: hard pellets ] & I $(n=10)$ & $44.1 \pm 12.9$ & $44.2 \pm 12.7$ & $44.4 \pm 12.6$ \\
& II $(n=9)$ & $2.9 \pm 3.7$ & $2.4 \pm 3.9$ & $2.8 \pm 4.2$ \\
& IV $(n=8)$ & $2.3 \pm 2.6$ & $2.0 \pm 2.7$ & $1.9 \pm 3.2$ \\
\hline
\end{tabular}

Group I: $0.75 \mathrm{~g} / \mathrm{d}$ of inulin during supplementation, Group II: $1.00 \mathrm{~g} / \mathrm{d}$ of inulin; Group III: $1.25 \mathrm{~g} / \mathrm{d}$ of inulin. Group IV: $0 \mathrm{~g} / \mathrm{d}$ of inulin. All data are given as the average \pm SD. * Significant changes as compared to basal period $(p<0.05)$ during inulin supplementation. 
a significant reduction in golden stool during the washout period. Infants not given any inulin showed no significant change in stools colour profile.

The 8 infants in the control group showed no changes in faecal characteristics. Other than the conditions described above, inulin showed no other observable effects on the general conditions (such as temperature, appetite or weight gain) of the subjected infants.

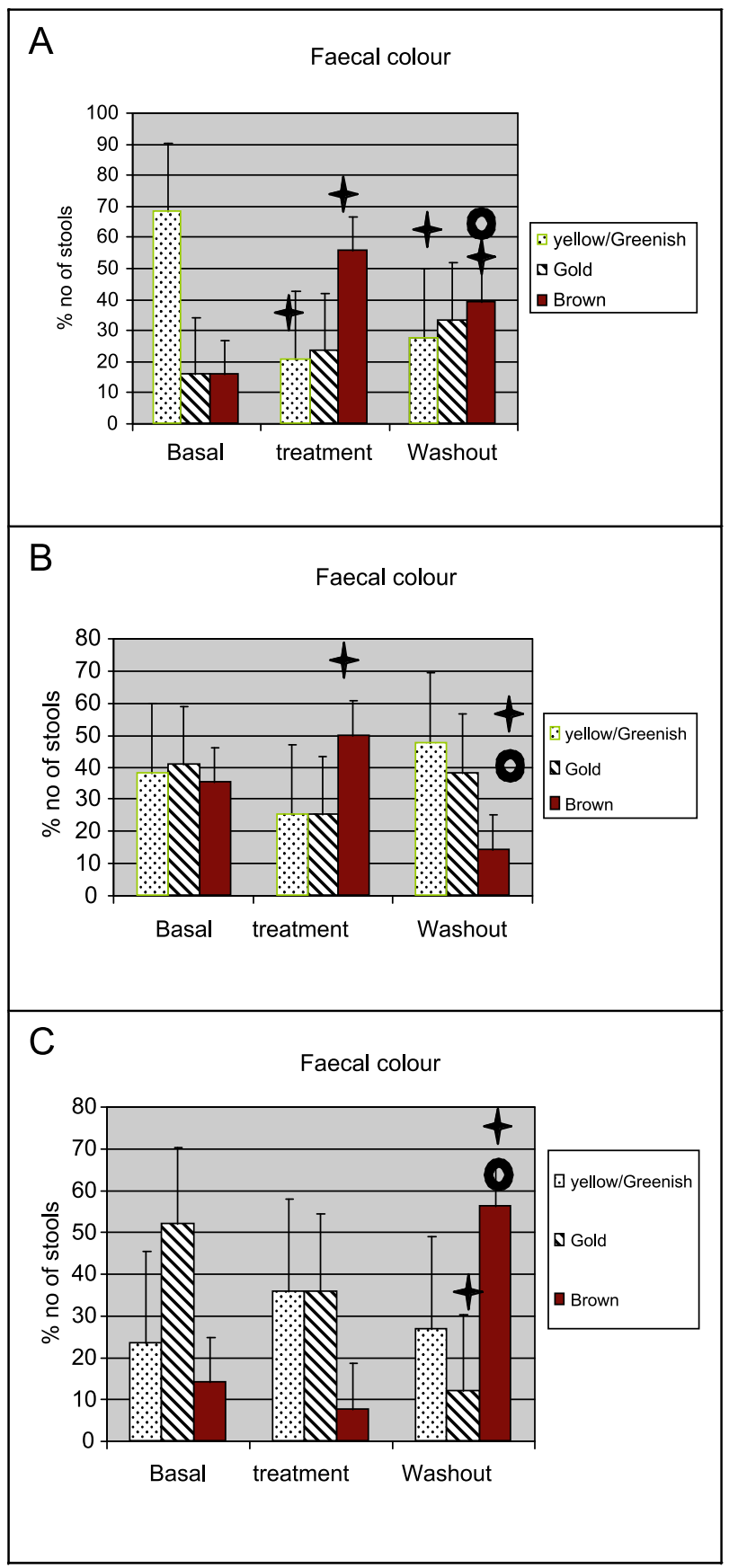

\section{Discussion}

It is known that there are significant differences in the colonic microbiota of breast-fed and formula-fed infants. The colon of breast-fed infants tend to be dominated by bifidobacteria, whereas those of formula-fed infants tend to have more diverse and adult like microflora [21, 22]. The present study also demonstrated a similar trend of microflora distribution. The faecal microbiota was dominated by bacteria such as coliforms, Gram-positive cocci and clostridia (Table 1). Low numbers of Lactobacillus spp. and Bifidobacterium spp. were observed in the faecal flora of these infants, and the highest inulin supplementation studied $(1.25 \mathrm{~g} / \mathrm{d})$ led to a significant increase in bifidobacteria. The present study also showed that with the lower levels of inulin supplementation the number of putrefactive bacteria could be reduced by almost one log unit. At dosages of 0.75 and $1.00 \mathrm{~g} / \mathrm{d}$ no significant increase in Bifidobacterium spp. was found.

The weak stimulation of bifidobacteria growth observed in this study may be explained by the fact that the stimulation effect of inulin or FOS is strain-specific [23, 24]. Bifidobacterium isolates collected from healthy infants in Serdang, Malaysia mainly belong to the species $B$. pseudocatenulatum [25]. Among the bifidobacteria strains investigated, B. pseudocatenulatum demonstrated only weak growth on inulin in vitro $[24,26]$. In vitro studies have demonstrated that $B$. longum is unable to ferment long chain polymers such as inulin efficiently, whereas $B$. adolescentis is able to metabolize inulin slightly better [24].

Fig. 2. Faecal colour during basal, treatment and washout periods in the various infants given different levels of inulin supplementation.

A: Infants treated with $0.75 \mathrm{~g} /$ day inulin showed an increase in brown stools and a reduction in yellow/greenish stool during treatment period. During the washout period, the number of brown stools decreased, while that of the yellow/greenish stool increased again. Infants not given any inulin showed no significant change in stools colour profile.

B: Infants treated with $1.00 \mathrm{~g} /$ day inulin also showed increase in the number of brown stools and a reduction in yellow/greenish stool during treatment. Again, during the washout period, the number of brown stools decreased, while that of the yellow/greenish stool increased.

$\mathrm{C}$ : Infants treated with $1.25 \mathrm{~g}$ /day inulin showed insignificant changes in stools colour during treatment, followed by a significant increase in brown stools and a significant reduction in golden stool during the washout period. Dagger: Significantly different $(p<0.05)$ from basal Open circle: Significantly different $(p<0.05)$ from treatment 
Another factor explaining the moderate changes found in this study may be the low daily dosage of inulins. For prebiotic effects from native inulin to be observed in adults at least $0.07 \mathrm{~g} / \mathrm{kg} /$ day is required [9]. The lowest prebiotic dosage reported in formula-fed infants (aged on average 12 weeks) is $1.5 \mathrm{~g} / \mathrm{d}$ or $0.25 \mathrm{~g} / \mathrm{kg} /$ day [19]. The dosage applied in our study $(0.1-0.16 \mathrm{~g} / \mathrm{kg} / \mathrm{d})$ was at the lowest part of this range. With oligofructose from inulin at $2 \mathrm{~g} / \mathrm{d}$, only a trend for increased bifidobacteria in children aged 6-24 months were found [27]. In paediatric patients it was found that a mixture of FOS and inulin at $0.8 \mathrm{~g} / \mathrm{d}$ led to a trend for bifidogenic effect [28]. Our data do not deviate significantly from these publications. Finally it may well be that the duration of the trial is relevant, two weeks of treatment being too short to pick up the changes.

The original number of bifidobacteria present before inulin supplementation may also play a role. Human studies showed no clear dose-response effect on the increase of log number of bifidobacteria [29,30], and it is hypothesized that the major factor in determining the increase in the amount of bifidobacteria is the initial number of bifidobacteria in the faeces at the start of the inulin feeding regimen. With the numbers already fairly high, an increase (combined with a low daily dosage) may be difficult to detect, and then only with the highest dosage applied here.

The decrease in faecal $\mathrm{pH}$ observed in this study indicated the production of SCFA and lactate through fermentation of inulin [31]. Stool begins green and changes to bright yellow as it passes through the digestive system. Bile and bacteria finally turn it brown. Yellow or green stools may indicate that the stool is passing through the digestive tract too quickly. Bright yellow stools may indicate insufficient bile [32]. The increase in number of brown stools of 0.75 and $1.00 \mathrm{~g} / \mathrm{d}$ inulin supplementation period indicated an improvement in the healthy bile production and bacterial fermentation. The reasons why infants treated with $1.25 \mathrm{~g} /$ day inulin showed insignificant changes in stool colour during treatment, but a significant increase in healthy brown stools and a significant reduction in golden stool during the washout period, is difficult to explained. The beneficial effects appeared to have been delayed by the high inulin dose, and only occurred during the washout period.

This study also showed changes in stool frequency and consistency during inulin supplementation in formula-fed children. However, none of these reached statistical significance. Similar data have been published for the consumption of FOS in children aged 32 weeks [33].

All data taken together in this study shows that the consumption of native inulin in children aged about 8 months at $1.25 \mathrm{~g} / \mathrm{d}$ leads to a prebiotic effect similar to that observed in adults: a significant increase in the number of faecal bifidobacteria accompanied by a decrease in potentially harmful species such as clostridia.

\section{Acknowledgement}

The researchers would like to thank paediatrician Dr Nur Atiqah A M from Pantai Medical Centre, for her advice at the beginning of the research and in obtaining the human ethics committee approval; and to Sensus Netherlands for sponsoring the research.

\section{References}

[1] Van Loo, J., Coussement, P., Leenheer, L.D., Hoebregs, H., and Smits, G.: On the presence of inulin and oligofructose as natural ingredients in the Western diet. Crit. Rev. Food Sci. Nutr., 35, 525-552, 1995.

[2] Campbell, J.M., Bauer, L.L., Fahey, Jr. G.C., Hogarth, A.J.C.L., Wolf, B.W., and Hunter, D.E.: Selected fructooligosaccharide (1-ketose, nystose, and 1f- $\beta$ fructofuranosylnystose) composition of foods and feed. $J$. Agric. Food Chem., 45, 3076-3082, 1997.

[3] Roberfroid, M. and Slavin, J.L.: In: Resistant Oligosaccharides. Handbook of Dietary Fiber. Marcel Dekker, Inc., Basel, New York, pp. 125-145, 2001.

[4] Gibson, G.R. and Roberfroid, M.B.: Dietary modulation of the human colonic microbiota: Introducing the concept of prebiotics. J. Nutr., 125, 1401-1412, 1995.

[5] Gibson, G.R., Beatty, E.B., Wang, X., and Cummings, J.H.: Selective stimulation of bifidobacteria in the human colon by oligofructose and inulin. Gastroenterology, 108, 975-982, 1995.

[6] Kruse, H.P., 22 Kleessen, B., and Blaut, M.: Effects of inulin on fecal bifidobacteria in human subjects. Br. J. Nutr., 82, 375-382, 1999.

[7] Menne, E., Guggenbuhl, N., and Roberfroid, M.: Fn-type chicory inulin hydrolysate has a prebiotic effect in humans. $J$. Nutr., 130, 1197-1199, 2000.

[8] Tuohy, K.M., Finlay, R.K., Wynne, A.G., and Gibson, G.R.: A human volunteer study on the prebiotic effects of HPinulin-faecal bacteria enumerated using fluorescent in situ hybridisation (FISH). Anaerobe., 7, 113-118, 2001.

[9] Kolida, S., Meyer, P.D., and Gibson, G.R.: A double blind placebo controlled study to establish the bifidogenic dosage of inulin in healthy humans. Eur. J. Clin. Nutr., 61, 1189$1195,2007$.

[10] Wang, X. and Gibson, G.R.: Effects of the in vitro fermentation of oligofructose and inulin by bacteria growing in the human large intestine. J. Appl.Bact., 75, 373-380, 1993.

[11] Meyer, D. and Stasse-Wolthuis, M.: Inulin and bone health. Curr. Top. Nutraceutical Res., 4, 211-226, 2006.

[12] Rafter, J., Bennett, M., Caderni, G., Clune, Y., Hughes, R., Karlsson, P.C., Klinder, A., O'Riordan, M., O’Sullivan, G.C., Pool-Zobel, B., Rechkemmer, G., Roller, M., Rowland, I., Salvadori, M., Thijs, H., van Loo, J., Watzl, B., and Collins, J.K.: Dietary synbiotics reduce cancer risk factors in polypectomized and colon cancer patients. Am. J. Clin. Nutr., 85, 488-496, 2007.

[13] Beylot, M.: Effects of inulin-type fructans on lipid metabo- 
lism in man and in animal models. Br. J. Nutr., 93, S163S168, 2005.

[14] Archer, B.J., Johnson, S.K., Devereux, H.M., and Baxter, A.L.: Effect of fat replacement by inulin or lupin-kernel fibre on sausage patty acceptability, post-meal perceptions of satiety and food intake in men. Br. J. Nutr., 91, 591-599, 2004.

[15] Cani, P.D., Joly, E., Horsmans, Y., and Delzenne, N.M.: Oligofructose promotes satiety in healthy human: a pilot study. Eur. J. Clin. Nutr., 60, 567-572, 2006.

[16] Seifert, S. and Watzl, B.: Inulin and oligofructose: review of experimental data on immune modulation. J. Nutr., 137, S2563-2567, 2007.

[17] Knol, J., Scholtens, P., Kafka, C., Steenbakkers, J., Gro, S., Helm, K., Klarczyk, M., Schopfer, H., Bockler, H.-M., and Wells, J.: Colon microflora in infants fed formula with galacto- and fructo-oligosaccharides: more like breast-fed infants. J. Pediatr. Gastroenterology and Nutr., 40, 36-42, 2005.

[18] Bakker-Zierikzee, A.M., Alles, M.S., Knol, J., Kok, F.J., and Tolboom, J.J.M.: Effects of infant formula containing a mixture of galacto- and fructo-oligosaccharides or viable Bifidobacterium animalis on the intestinal microflora during the first 4 months of life. Br. J. Nutr., 94, 783-790, 2005.

[19] Kim, S.-H., Lee, D.H., and Meyer, D.: Supplementation of baby formula with native inulin has a prebiotic effect in formula-fed babies. Asia Pac. J. Clin. Nutr., 16, 172-177, 2007.

[20] Cheesbrough, M.: Medical laboratory manual for tropical countries (Volume II). Tropical Health Technology, Butterworth Scientific Ltd, Cambridgeshire, pp. 294-299, 1984.

[21] Harmsen, H.J.M., Wildeboer-VeLoo, A.C.M., Raangs, G.C., Wagendrop, A.A., Klijn, N., Bindels, J.G., and Welling, G.W.: Analysis of intestinal flora development in breast-fed and formula-fed infants by using molecular identification and detection methods. J. Pediatr Gastroenterology Nutr., 30, 61-67, 2000.

[22] Mitsuoka, T.: Prebiotics and intestinal flora. Biosci. Microflora, 21, 3-12, 2002.
[23] Roberfroid, M.B.: Prebiotics: preferential substrates for specific germs? Am. J. Clin. Nutr., 73, 406S-409S, 2001.

[24] Rossi, M., Corradini, C., Amaretti, A., Nicolini, M., Pompei, A., Zanoni, S., and Matteuzzi, D.: Fermentation of fructooligosaccharides and inulin by bifidobacteria: a comparative study of pure and fecal cultures. Appl. Environ Microbiol., 71, 6150-6158, 2005.

[25] Shuhaimi, M., Ali, A.M., Saleh, N.M., and Yazid, A.M.: Classification of Bifidobacterium isolates from infant faeces using PCR-based and 16S rDNA partial sequences analysis methods. Biosci. Microflora, 21, 155-161, 2002.

[26] Bielecka, M., Biedrzycka, E., and Majkowska, A.: Selection of probiotics and prebiotics for synbiotics and conformation of their in vivo effectiveness. Food Res. Int., 35, 125-131, 2002.

[27] Waligora-Dupriet, A.-J., Campeotto, F., Nicolis, I., Bonet, A., Soulaines, P., Dupont, C., and Butel, M.-J.: Effect of oligofructose supplementation on gut microflora and wellbeing in young children attending a day care centre. Int. J. Food Microbiol., 113, 101-113, 2007.

[28] Zheng, S., Steenhout, P., Kuiran, D., Qihong, W., Weiping, W., Hager, C., Haschke, F., and Clemens, R.A.: Nutritional support of pediatric patients with cancer consuming an enteral formula with fructooligosaccharides. Nutr. Res., 26, 154-162, 2006.

[29] Rao, V.A.: The prebiotic properties of oligofructose at low intake levels. Nutr. Res., 21, 843-848, 2001.

[30] Roberfroid, M.B., Van Loo, J.A.E., and Gibson, G.R.: The bifidogenic nature of inulin and its hydrolysis products. $J$. Nutr., 128, 11-19, 1998.

[31] Yap, K.W., Mohamed, S., Yazid, A.M., and Meyer, D.M.: Dose-response effects of inulin on the faecal short-chain fatty acids content and mineral absorption of formula-fed infants. Nutr. \& Food Sci., 35, 208-219, 2005.

[32] Defelice, K.: www.enzymestuff.com/rtstools.htm, 2002.

[33] Moore, N., Chao, C., Yang, L.-P., Storm, H., Oliva-Hemker, M., and Saavedra, J.M.: Effects of fructo-oligosaccharidesupplemented infant cereal: a double-blind, randomized trial. Br. J. Nutr., 90, 581-587, 2003. 